\title{
N $86-23498$
}

\section{LABORATORY STUDIES OF INTERPLANETARY DUST}

\author{
R. M. Walker \\ MeDonnell Center for the Space Seiences \\ and \\ Department of Physies \\ Washington University \\ St. Louis, MO 63130, USA
}

\author{
Report prepared for: \\ Workshop in the Interrelationships Among Circumstellar. \\ Interstellar, and Interplanetary Cirains, \\ Fobruary 17, 1985, Wye. Maryland
}




\section{LABORATORY STUDIES OF INTERPLANETARY DUST}

\section{INTRODUCTION}

Interplanetary dust particles collected in three different ways are now available for laboratory study. Impact collectors flown on aircraft sampling the stratosphere have provided the most important source of (more or less) unaltered interplanetary dust material [1]. Larger cosmic particles have been recovered from sediments, both from the sea [2], and, most recently, from temporary glacial lakes in Greenland [3]. Finally, debris samples from impacting interplanetary particles have also been collected in experiments flown in earth orbit and returned to earth $[4,5$, and $6 \mathrm{~b}] . *$

This brief review treats only the analysis of dust particles col'ected in the stratosphere. These particles are the best available samples of interplanetary dust and have been studied using a variety of analytical techniques. The particles are systematically collected and curated by a group at the Johnson Space Center, Houston. Catalogs of the collections have been published and individual particles are made available to qualified investigators on request [8].

The stratospheric dust particles have been called by a variety of names (including the deserved description as "Brownlee particles") and a word about nomenclature is in order. In what follows, stratospheric particles whose major element compositions are similar to those of chondritic meteorites, are referred to as "interplanetary dust particles or IDPs." Some particles in this chemical class are demonstrably extraterrestrial. To facilitate discussion, certain IDPs have also been given individual names, e.g. Calrissian, Skywalker, etc. Particles in other chemical classes are not treated in this paper. Although some of them may be extraterrestrial, both man-made and natural contaminants are abundant in the non-chondritic chemical classes.

For a more complete view of IDPs and other cosmic dust particles the reader is referred to two revicus $[9,10]$.

The following questions are addressed in this workshop paper:

1. Is it certain that chondritic stratospheric dust particles are extraterrestrial?

2. What are the general physical, chemical, mineralogical, isotopic and infrared properties of IDPs?

3. How do IDP's compare with other extraterrestrial materials, specifically unequilibrated meteorites and comet dust?

4. DoIDPs consist of primitive and/or primordial matter?

5. Is there any relationship between IDPs and interstellar matter?

6. Can the study of IDPs contribute to the understanding of basic astrophysical problems?

e.g.: the conditions under which solids form in space?

Some of these questions have clear answers, others not. Nor, are the questions independent of each other.

* In Ract. there is currently a spacecraft that has been in near-earth orbit since April of 1981 (the Long Duration Exposure Facility - LDEF I), which has several dust collection experiments on board. Griginally scheduled to return to earth in March of 1985, the recovery has now been delayed. Just in the last fer weeks. portions of a thermal blanket returned by astronauts from the holar Slax Repair Mlimion have been found to contsin imparts of likely extraterrestrial origin [6b].

This impas debris material will shortly be made avalable for general scientific study [7|. 


\section{IS IT CERTAIN THAT CHONDRITIC STRATOSPHERIC DUST PARTICLES ARE EXTRATERRESTRIAL?}

Yes. However, many early studies of purported interplanetary dust particles were erroneous, and it is useful to review the evidence bearing on this fundamental point.*

The simplest proof that IDPs are extraterrestrial is the recent observation of large densities of fossil nuclear particle tracks in silicate crystals contained within IDPs [12]. Massive nuclear particles $(Z \geq 20)$ are needed to produce tracks in silicates. In the terrestrial environment the only natural source of such nuclear particles is the spontaneous fission of ${ }^{238} \mathrm{U}$, and even in old terrestrial samples, fission track densities are low in silicates. In contrast, samples of extraterrestrial silicates, such as lunar soil grains, typically show high track densities produced by energetic heavy nuclei from the sun and by galactic cosmic rays a complete discussion of fossil nuclear tracks is given in Reference 13].

The extraterrestrial origin of IDPs had been firmly established even before the recent track observations. Ion probe measurements demonstrated that some IDPs are highly enriched in deuterium relative to hydrogen compared to terrestrial samples [14a]. Still earlier measurements $[15,16]$ showed that the particles possessed nonterrestrial abundances and compositions of noble gases. The fact that IDPs are chondritic in composition is strong evidence in itself of an extraterrestrial origin.

Although the presence of tracks or a large D/H anomaly can be considered as proof that a particular dust particle has resided in interplanetary space, the absence of these effects does not preclude an extraterrestrial origin. It is thus impossible to be certain that all particles labelled IDPs are micrometeorites; however, this is a plausible working hypothesis.

\footnotetext{
* An excellent description of the early confused state-of-affairs is given by llodge in his recent book 11: At one point. there was general agrement between a priori entimates of interplanetary dust influx. particle collections performed with high altitude balloons, and data from electronic detectors in space. luforimately, these were all in error by a very large factor, Interplanetary dust collertion proved a more formidable probiem than early investigators realized.
} 


\section{WHAT ARE THE PHYSICAL, MINERALOGICAL, ISOTOPIC AND INFRARED PROPERTIES OF IDPA?}

IDPs are an extremely diverse set of objects. It is beyond the scope of this paper to describe all the observations that have been made. The general kinds of measurements that are possible will be briefly described and some particular features will be highlighted.

In spite of their small masses $\left(\sim 10^{-8}\right.$ to $\left.10^{-10} \mathrm{gms}\right)$ it has proven possible to develop a variety of techniques for studying individual particles. Initial examination in a scanning electron microscope (SEM) equipped with an X-ray detection system (EDX) reveals the morphologies and major element compositions. Particles can then be individually weighed and their densities determined, although this has been done in only a few cases [17]. More typically, a particle is crushed or otherwise dispersed and parts of it are transferred to an electron microscope grid where detailed measurements of structures and mineralogies can be made by transmission electron microscopy (TEM) [18a-q]. The bulk of the crushed particle can be transferred to a $\mathrm{KBr}$ crystal for measurement of its infrared transmission spectrum [19]. If the particle is subsequently mounted on a gold substrate isotopic measurements on different parts of a particle are possible using an ion probe [14a-d]. Alternatively, the particle can be totally consumed in a thermal ionization source mass spectrometer to obtain precision isotopic measurements of several selected elements, or an ensemble of particles can be vaporized in a noble gas mass spectrometer to obtain elemental and isotopic information on noble gases $\{15,16\}$. Micro-Raman techniques have been used to establish the presence of "disordered graphite" in at least one particle [34] and Auger spectroscopy has been used on two non-chondritic stratospheric dust particles to look for evidence of surface contamination due to interaction with atmospheric aerosols [35].

Most measurements have been reported by groups specializing in one kind of instrumentation or another, and in only a few cases have the same particles been studied by a variety of techniques. Increasingly, however, sequential or parallel measurements of different types are being made on single particles.

Many IDPs consist of extremely porous aggregates with a typical "cluster of grapes" morphology [9]. Indeed, the erroneous belief has arisen that all IDPs consist of low density, lluffy materials. This is not true. Although all IDPs consist of aggregates of material in the sense that they are not fragments of an igneous rock or pieces of a molt droplet, many are quite compact and have densities of $\sim 2 \mathrm{gms} / \mathrm{cm}^{3}$ or higher [17].

Detailed measurements of the mineralogies and structures of about two dozen particles have been reported [18a-q]. Some particles consist of collections of small anhydrous crystalline grains which are imbedded in an amorphous carbonaceous material. The proportion of amorphous material is highly variable from one particle to the next. The minerals olivine and pyroxene are common, as are magnetite and sulfides of different types. The proportions of different mineral phases vary from particle to particle as do the morphologies and compositions of a given mineral type. Microchondrules, flattened where they are in contact with one another, have been observed, as have lath-like whiskers of pyroxene. Still another large class of particles contain phyllosilicates and give evidence of interaction with water.

A first order result of these studies is that different particles have qualitatively different assemblages of minerals. IDP's cannot be lumped together as a single class of objects; they must be studied individually.

Although infrared transmission measurements were undertaken to provide data that could be compared with astronomical observations, they have also provided a relatively quick means of measuring the dominant silicate mineralogy of whole IDPs. Most IDPs fall into one of three 
IR spectral classes called "olivine," "pyroxene," or "layer lattice silicate" from the similarity of the $10 \mu \mathrm{m}$ features with those of terrestrial mineral standards [19]. Complementary detailed TEM measurements on several particles in the different spectral classes confirm the IR designations. However, the detailed structures seen in the TEM are different for particles in the same spectral class, underlining the importance of treating IDPs as individual objects.

IR spectroscopy can also be used to locate particles with unusual mineralogies. For example, Calrissian, a particle in the layer lattice silicate class, has an atypically strong absorption at $6.8 \mu \mathrm{m}$ and an accompanying weaker absorption at $11.4 \mu \mathrm{m}$. These spectral features were interpreted as evidence for carbonates [19] and subsequent TEM measurements have confirmed the presence of numerous grains of $\mathrm{Fe}$ and $\mathrm{Mg}$ carbonate $[18 \mathrm{~m}]$.

Ion probe isotopic measurements show large, but variable deuterium enrichments in 5 of 8 IDPs measured to date [14]. Maximum enrichments of $\sim 250 \%$ are seen. In particles with deuterium enhancements the $\mathrm{D} / \mathrm{H}$ values are variable on the scale of a few microns. Correlation of the isotopic signatures with other ion signals indicate that the deuterium excess is associated with $\mathrm{C}$ but not with $\mathrm{OH}[14 \mathrm{~b}]$. In contrast, carbon isotopic ratios are found to be constant from one part of a particle to the next although differences between particles are found [14c]. Both $\mathrm{Mg}$ and $\mathrm{Si}$ give constant isotopic ratios consistent with terrestrial values in three particles [14c]. Earlier, higher precision measurements on several IDPs made using a thermal ionization source mass spectrometer indicated the possible presence of $\mathrm{Mg}$ isotopic anomalies at the level of up to $0.4 \%[20]$. 


\section{HOW DO IDPs COMPARE WITH METEORITES AND COMETS?}

Similar in some ways, apparently different in others. Consider first the comparison of IDPs with the fine-grained matrix material of unequilibrated meteorites. The question that dominated the original studies of IDPs was whether they were simply smaller versions of the larger, better known carbonaceous meteorites. No fragments resembling the porous, fluffy IDPs have yet been found in meteorites. It quickly became evident that IDPs were a unique form of extraterrestrial material, different from carbonaceous chondrites and deserving of detailed study in their own right. Moreover, there are distinctive differences in the detailed mineralogies seen in both porous and compact IDPs and the mineralogies observed in meteorites. For example, although CM carbonaceous chondrites and one IR class of IDPs have similar IR spectra dominated by phyllosilicates, the detailed structures of the phyllosilicates are different [18h].

Given the initial question, most authors have emphasized the differences between IDPs and meteorites. Yet there are strong similarities e.g.: the deuterium enrichments seen in IDPs, acid residues of carbonaceous meteorites, and matrix materials from some unequilibrated chondrites. It is not clear whether the differences between IDPs and meteorites are fundamental or whether they represent differences in degree, rather than in kind. It must be remembered that meteorites represent a subset of objects that survive atmospheric entry. There may be large objects, consisting of assemblages of material similar to IDPs, that never reach earth because of their fragility.

At this point it would appear prudent to reserve judgement on the relationship between IDPs and meteorites and treat them as related parts of the same larger puzzle.

Whether true or not, there is a widely held belief, based largely on mass balance arguments, that interplanetary dust is composed primarily of comet dust [23]. It is also believed by many that comets consist largely of primordial material. Direct comparison of IDPs with cometary material suffers from the obvious problem that no one has yet mounted a space mission to return a comet sample.

In the absence of a returned comet sample, the only direct comparison between IDPs and comet dust is based on their optical properties [19]. The best astronomical observations in the infrared are those for Comet Kohoutek where the spectral emission properties of the dust have been obtained from deconvolution of the observational data using an assumed black body spectrum [36]. Comparison with the spectral transmission data for IDPs shows that none of the spectral classes of the particles gives a good match to the comet result. However, a composite spectrum consisting of equal contributions from the pyroxene and layer-lattice silicate classes gives a reasonable match. The olivine spectral class gives the worst fit and it appears unlikely that many particles in this class (some of which are known to be extraterrestrial) are present in Comet Kohoutek.

The present results therefore suggest either that the olivine component of interplanetary dust is not derived from comets or that Comet Kohoutek is not representative of all comets. The olivine class of particles may also be over represented in the limited population of particles (a total of 26) so far measured. These possibilities are obviously not mutually exclusive.

Spectral matching is not a very satisfactory way to approach the question of the relationship between IDPs and comets since even a perfect spectral match would not guarantee that IDPs came from comets. The main thing in its favor is that it is currently the only way to attack the problem.

Apart from obtaining additional, sorely needed spectral data on both comet dust and IDPs, there are several experimental approaches to solving the IDP-comet comparison problem. It has 
long been known that most meteor showers have orbits that link them directly with specific comcts. Collection and analysis of material from a meteor shower would be a major accomplishment, and a joint Franco-Soviet space experiment which will attempt to collect impact debris from particles associated with the comet Giacobini-Zinner is currently in progress [21].

As discussed more fully elsewhere [25], another important approach would be to construct, a space instrument that would measure the orbital parameters of individual particles whose impact debris atoms would be analyzed upon return to the laboratory. Interstellar grains traversing the solar system conceivably could also be located and measured with such an instrument.

Comet rendezvous missions performing sophisticated in situ measurements on dust grains would also help settle the question. And, of course, a sample return mission to a comet would be invaluable.

Scientific interest in IDPs would likely be enhanced if comet samples proved to contain particles of a similar nature. At best, space missions can sample only a few comets in the foreseeable ruture. If IDPs do indeed come predominantly from comets they are samples of many different objects; further, their heterogeneity shows that they contain a record of a varicty of processes. 


\section{DO IDP\& CONSIST OF PRIMITIVE AND/OR PRIMORDIAL MATTER?}

Yes and maybe. No generaliy accepted criteria exist to classify material as either primitive or primordial. For the purposes of this paper, primitive matter is defined as material which has isotopic struc* ures different from those in "average solar system material" and of a nature that cannot be explained by known processes such as spallation reactions by cosmic rays during exposure in interplanetary space. The implication is that primitive materials can be used to obtain information about conditions in the early solar system.

Primordial matter can be defined as (more or less) unaltered material that existed in the (presumed) gas-dust cloud that existed the prior to the present solar system i.e.: interstellar dust. Certain authors have interpreted isotopic data as indicating the presence of such material in meteorites [21]. However, the evidence is circumstantial; no one to date has isolated specific meteoritic grains that have been definitively identified as unaltered interstellar material.

One of the most intriguing of the many isotopic anomalies that have been discovered in meteorites in recent years in the case of $\mathrm{Ne}-\mathrm{E}$. This component of Ne consists almost exclusively of ${ }^{22} \mathrm{Ne}[22]$. A plausible explanation of $\mathrm{Ne}-\mathrm{E}$ is that live ${ }^{22} \mathrm{Na}$ was incorporated into solid grains where it subsequently decayed into ${ }^{22}$ Ne. Preservation of the isotopic signature would require that the solid carrier phases were never heated to the point where they totally degassed. Thus they might still be present in recognizable form. However, it remains to be proven that this is the case.

Primordial matter may well exist in both meteorites and IDPs. However, it may be more difficult to identify such material in IDPs simply because of their small sizes. More detailed studies using improved analytical techniques and working with larger IDPs (a program to collect larger stratospheric dust particles is currently in progress) will help address this question. Other aspects of the relationship of IDPs to interstellar matter are treated in the next section. 


\section{IS THERE ANY RELATIONSHIP BETWEEN IDPs AND INTERSTELLAR MATTER?}

Maybe, maybe not. The most plausible place to look for such links is in dense gas-dust clouds containing strong infrared sources that have been interpreted as protostars. A comparison of the IR absorption features of the protostar W33A with the spectrum of a typical IDP of the layer lattice silicate class shows certain common features. Present in both spectra are features at $3 \mu \mathrm{m}, 6.8 \mu \mathrm{m}$ and $10 \mu \mathrm{m}$. The dominant $3 \mu \mathrm{m}$ feature in $W 33 \mathrm{~A}$ has been attributed to water ice, a constituent that cannot be present in IDPs where the weaker $3 \mu \mathrm{m}$ feature is attributable to water incorporated in a layered silicate structure. The $10 \mu \mathrm{m}$ feature seen in both spectra is likely due to silicates.

In the IDPs the $6.8 \mu \mathrm{m}$ feature is certainly primarily due to carbonate minerals, although a smaller contribution from other phases cannot be ruled out at this time. It is an open question whether carbonates are also responsible for the ubiquitous $6.8 \mu \mathrm{m}$ feature seen in $\mathrm{W} 33 \mathrm{~A}$, and other protostellar objects. Such a possibility was originally suggested based on a comparison of the IR spectrum of the meteorite Murchison with protostellar spectra [26]. However, the absence of an expected accompanying carbonate feature at $>25 \mu \mathrm{m}$ [27] was also noted. In principle, the presence of carbonate minerals in protostellar sources can be settled by additional astronomical measurements.

Another possible connection between IDPs and interstellar cloud material is provided by the deuterium results. Radio observations show that simple molecules such as HCN can be greatly enriched in deuterium in cold interstellar clouds [28a,b]. If interstellar grains are partly composed of complex organic molecules built up from simple molecules by processes such as photolysis $[29]$, it is plausible that the complex molecules would reflect the deuterium enrichments present in the simple precursor molecules. The large deuterium enrichments seen in IDPs (and in certain extracts from unequilibrated meteorites) could be due to such deuterated interstellar material [30].

Although it is useful to consider points of contact between IDPs and interstellar material, it is equally important to consider what has not been observed. The depletions of certain elements (e.g.: $\mathrm{Al}$ and $\mathrm{Ca}$ ) in the interstellar gas phase have led to the view that interstellar grains in clouds should consist in part of refractory cores [31]. No such refractory cores have yet been reported in IDPs, although fassaite, a common constituent of calcium aluminum rich refractory inclusions in meteorites has been recently found in one IDP $[18 \mathrm{k}$.

That grains with the properties expected for interstellar material have not yet been reported in IDPs, does not necessarily mean that such material is not present. Perhaps the problem is one of concentration and scale. Most of the astronomical observations relate to particles of $\sim 0.1 \mu \mathrm{m}$ or less. While grains of this size are routinely measured in electron diffraction studies of IDPs, detailed TEM measurements tend to be made on the largest subgrains, on those which have euhedral external morphologies, and those which, by definition, give the strongest electron diffraction signals. Many sub-grains of the kind inferred from astronomical measurements could be present as constituents of modest crystallinity without having been singled out in the experimental studies done to date. 


\section{CAN THE STUDY OF IDPs CONTRIBUTE TO THE UNDERSTANDING OF BASIC ASTROPHYSICAL PROBLEMS?}

Probably - especially on the question of the formation of solids in space. Wherever and whenever the various components of IDPs were formed, several lines of evidence indicate that vapor-solid processes were important. Crystal morphologies unlike those found in terrestrial, lunar, or meteoritic rocks are a common feature in IDPs. Specifically, in fluffy, anhydrous particles the mineral pyroxene is observed to occur in the form of whiskers (rods and ribbons) and very thin platelets. The presence of axial screw dislocations and absence of extensive twinning have been used to argue that the crystals were formed by direct vapor phase condensation [18e]. It is interesting that the results of laboratory experiments on crystal growth served to predict the presence of the observed morphologies prior to their observation in nature $[32]$.

Mineralogical and morphological features found in other anhydrous porous aggregates have been interpreted as evidence for the formation of carbon compounds by heterogeneous catalytic reduction of carbon-rich gases $[18 \mathrm{f}, 18 \mathrm{~g}]$. Carbonaceous mantles filaments and fine-grained matrix material are found intermixed with $\mathrm{Fe}-\mathrm{Ni}$ grains and $\mathrm{Fe}-\mathrm{Ni}$ carbides of several types, notably hexagonal $\epsilon$-carbide. Similar phases are characteristic by-products of the laboratory decomposition of $\mathrm{CO}$ by fine metal particles. Such catalytic processes are widely used industrially with an important subset being the Fischer-Tropsch reactions. Based largely on the observed distribution of molecular weights of extracted hydrocarbons, such processes had been previously proposed as a mechanism for the formation of organic compounds in meteorites [33].

Although these results are intriguing, the lack of detailed knowledge of the kinetics of catalytic reactions in plausible astrophysical environments currently limits the constraints that can be put on the physical conditions under which the observed structures were formed.

Although olivine and pyroxene platelets have been more commonly observed in anhydrous particles, they also occur in IDPs of the hydrated silicate type. In one case, a phyllosilicate crystal is observed in intimate association with a pyroxene crystal suggesting that phyllosilicates are produced by aqueous alteration of pyroxenes $[18-\mathrm{k}]$. Whether this was a liquid or vapor reaction is not clear. It has also been suggested that the carbonates observed in layer-lattice silicate IDPs may have been formed by catalytic reaction with silicates and magnetite in a cooling gas $[18-\mathrm{m}]$.

The ensemble of these mineralogical studies, coupled with the fact that many of the particles possess anomalous deuterium abundances, suggest that IPDs contain a record of processes going on early in the history of the solar system, or possibly, before. 


\section{CONCLUDING REMARKS}

IDPs are a unique form of primitive extraterrestrial material. Their systematic study is less than a decade old. In spite of the formidable experimental problems in working with particles that are too small to be seen with the naked eye, it has proven possible to obtain considerable information concerning their properties and possible origins. Like any new field, there are many unanswered questions. Continued work by a growing number of investigators, using ever more sophisticated analytic techniques, can be expected to give continued progress and almost certainly to produce some surprises. 


\section{REFERENCES}

1. Brownlee, D. E. Tomandl D. A., and Olszewski E. (1977) Interplanetary Dust; a new source of extraterrestrial material for laboratory studies. Proc. 8th Lunar Sci. Conf., p. 149-160.

2. Brownlee D. E. (1981) The Sea, Vol. 7, (ed., C. Emiliani), p. 733.

3. Brownlee, D. E., Fehrenbach L., Hammer C., Jehand C., Maurette M., and Thomsen H. H. (1985) A new mine of cosmic dust grains in the melt zones of the Greenland ice cap. Lunar Planet. Sci. XVI, Houston, TX, p. 95.

4. Clanton U.S., Zook H. A., and Schultz R. A. (1980) Hypervelocity impacts on Skylab N/Apollo windows. Proc. 11th Lunar Planet. Sci. Conf., p. 2261-2273.

5. McDonnell J. A. M., Carey W. C., Dixon D. G. (1984) Cosmic dust collection by the capture cell technique on the Space Shuttle. Nature 909, p. 237-240.

6a. Kessler D. J., Zook H. A., Potter A. E., McKay D. S., Clanton U.S., Warren J. L., Watts L. A., Schultz R. A., Schramm L. S., and Robinson G. A. (1985) Examination of return of Solar-Max surfaces for impacting orbital debris and meteoroids. Lunar and Planet. Sci. XVI, p. $434-435$.

6b. Schramm L. S., McKay D. S., Zook H. A., and Robinson G. A. (1985) Analysis of micrometeorite material captured by the Solar-Max Satellite. Lunar Planet. Sci. XVI, p. 736-737.

7. Investigators interested in studying impact material recovered from the Solar-Max Mission should contact D. S. McKay, NASA/Johnson Space Center, Houston, TX 77058.

8. Those interested in studying IDPs should contact M. E. Zolensky, SN4, NASA/Johnson Space Center, Houston, TX 77058.

9. Fraundorf P., Brownlee D. E., and Walker R. M. (1982) Laboratory studies of interplanetary dust. In Comets (L. Wilkening, ed.), Univ. of Arizona Press, p. 383-409.

10. Brownlee D. E., (1985) Cosmic dust: collection and research. Annual Review of Earth and Planetary Sciences. 19 (G. Wetherill, ed.).

11. Hodge, P. H. (1981) Interplanetary Dust (Gordon and Breach), New York.

12. Bradley J. P., Brownlee D. E., Fraundorf P. (1984) Discovery of nuclear tracks in interplanetary dust. Science 226, p. 1432-1434.

13. Fleischer R. L., Price P. B., and Walker R. M. (1975) Nuclear Tracks in Solids. (University of Calif. Press).

1.1a. Zinner E., Mckeegan K. D., and Walker R. M. (1983) Laboratory measurements of D/H ratios in interplanetary dust. Nature 305, p. 119-121. 
14b. Zinner E. and McKeegan K. D. (1984) Ion probe measurements of hydrogen and carbon isotopes in interplanetary dust. Lunar Planet. Sci. XV, Houston, TX, p. 961.

14c. Zinner E., Fahey r., and McKeegan K. D. (1984) Magnesium and silicon isotopic composition of interplanetary dust particles. Meteoritics 19, p. 345.

14d. An article summarizing the ion probe results and comparing them to other measurements on IDPs has been submitted to Geochim. Cosmochim. Acta, 1985.

15. Rajan R. S., Brownlee D. E., Tomandl D., Hodge P. W., Farrar H, and Britten R. A. (1977) Detection of ${ }^{4} \mathrm{He}$ in stratospheric dust particles gives evidence of extraterrestrial origin. Nature 267, p. 133-134.

16. Hudson B., Flynn G. J., Fraundorf P., Hohenberg C. M., and Shirck J. R. (1981) Noble gases in stratospheric dust particles: confirmation of extraterrestrial origin. Science 211 , p. $383-386$.

17. Fraundorf P., Hintz C., Lowry O., McKeegan K. D., and Sandford S. (1982) Determination of the mass, surface density and volume density of individual dust particles. Lunar Planet. Sci. XIII, Houston, TX, p. 225-226.

18a. Brownlee D. E. (1978) Interplanetary dust: possible implications for comets and pre-solar interstellar grains. In Protostars and Planets (T. Gehrels, ed.) Univ. of Arizona Press, p. 134.150 .

18b. Flynn G. J., Fraundorf P., Shirck J., and Walker R. M. (1978) Chemical and structural properties of "Brownlee" particles. Proc. 9th Lunar Planet. Sci. Conf., p. 1187-1208.

18c. Fraundorf P. and Shirck J. (1979) Microcharacterization of "Brownlee" particles: features which distinguish them from meteorites? Proc. 10th Lunar Planet. Sci. Conf., p. 951-976.

18d. Fraundorf P. (1981) Interplanetary dust in the Transmission Electron Microscope: diverse materials from the early solar system. Geochim. Cosmochim. Acta 45, p. 915-943.

18e. Bradley J. P., Brownlee D. E., and Veblen D. R. (1983) Pyroxene whiskers and platelets in interplanetary dust: evidence of vapour phase growth. Nature 301, p. 473-477.

18f. Christoffersen R. and Buseck P. R. (1983) Epsilon carbide: a low-temperature component of interplanetary dust particles. Science 229, p. 1327-1328.

18g. Bradley J. P., Brownlee D. E., and Fraundorf P. (1984) Carbon compounds in interplanetary dust: evidence for formation by heterogeneous catalysis. Science 223, p. $56-57$.

18h. Tomeoka K. and Buseck P. R. (1984) Transmission electron microscopy of the "Low-Ca" hydrated interplanetary dust particle. Earth Planet. Sci. Lett. 69, p. 243-254.

18i. Christoffersen R. and Buseck P. R. (1984) Mineralogy of platelet grains in carbon-rich interplanetary dust particles. Lunar Planet. Sci. XV, Houston, TX, p. 152-153. 
18j. Mackinnon I. D. R. and Rietmeijer F. J. M. (1984) Bismuth in interplanetary dust. Nature 311, p. 135-138.

18k. Tomeoka K. and Buseck P. R. (1984) Hydrated interplanetary dust particle linked with carbonaceous chondrites. Nature (in press). See also Lunar Planet Sci. XV, Houston, TX, p. 858 (1984).

181. Christoffersen R. and Buseck P. R. (1985) Mineralogy of the olivine class of interplanet:iry dust. Lunar Planet. Sci. XVI, Houston, TX, p. 127-128.

$18 \mathrm{~m}$. Tomeoka K. and Buseck P. R. (1985) Calrissian - a carbonate-rich hydrated interplanetary dust particle: possible residual material from protostellar clouds. Lunar Planet. Sci. XVI, Houston, TX, p. 862-863.

18n. McKay D. S., Rictmeijer F. J. M., and Mackinnon I. D. R. (1985) Mineralogy of chondritic prous aggregates: current status. Lunar Planet. Sci. XVI, Houston, TX, p. 536-537.

180. Rietmeijer F. J. M. (1985) Low-temperature aqueous and hydrothermal activity in a proto-planetary body: goethite, opal-CT, gillisite, and anatase in chondritic porous aggregate W7029. Lunar Planet Sci. XVI, Houston, TX, p. 696-697.

18p. Rietmeijer F. J. M. (1985) On the continuum between chondritic interplanetary dust and CI and CM chondrites: a petrological approach. Lunar Planet. Sci. XVI, Houston, TX, p. 698-699.

18q. Rietmeijer F. J. M. and Mackinnon I. D. R. (1985) A multistage history for carbonaceous material in chondritic porous aggregate W7029A and a new cosmothermometer. Lunar Planet. Sci. XVI, Houston, TX, p. 700-701.

19. Although short accounts of different aspects of the IR work on IDPs have been published in several places, a complete summary is given in a paper by S. Sandford and R. M. Walker, "Laboratory infrared transmission spectra of individual interplanetary dust particles from 2.5 to 25 microns" that will appear in the April 15, 1985 issue of the Astrophysical Journal.

20. Esat T. M., Brownlee D. C., Papanastassiou D. A., and Wasserburg G. J. (1979) The Mg isotopic composition of interplanetary dust particles. Science 208, p. 190-197.

21. For an example, see Swart P. K., Grady M. M., Pillinger C. T., Lewis R. S., and Anders E. (1983) Interstellar carbon in meteorites. Science 220, p. 406-410.

22. Eberhardt P., Jungck M. H. A., Meier F. O., and Niederer F. R. (1981) A neon-E rich phase in Orgueil: results obtained on density separates. Geochim. Cosmochim. Acta 45 , p. $1515-1528$.

23. For a recent discussion of this point see Whipple F. L. (1978) In Cosmic Dust (J. A. M. McDonnell, ed.) J. Wiley and Sons, New York, p. 1-70.

21. Bibring "J-P., Borg J., Langevin Y., Rosenbaum B., Vassent B., Solvetat P.. and Surkhov Y. A. (1985) Collection in space of cometary material by the KMP-Comet Experiment. 
Lunar Planet. Sci XVI, p. 55-56.

25. A discussion of instrumentation for future dust studies in space is given in an unpublished report entitled "LDEF II Cosmic Dust Experiments" available from R. Walker, Washington University, St. Louis, MO.

26. Knacke R. F. and Kr̈atschmer (1980) Infrared spectra of hydrated silicates, carbonaceous chondrites, and amorphous carbonates compared with interstellar dust absorptions. Astron. Astrophys. 92, p. 281-288.

27. Puetter R.C., Russell R. W., Soifer B.T. and Wilner S.P. (1979) Spectrophotometry of compact $H$ II regions from 4 to 8 microns. Astrophys. J. 228, p. 118.

28a. Penzias A. A. (1980) Nuclear processing and isotopes in the Galaxy. Science 208, p. $663-$ 669.

28b. Wannier P. G. (1980) Nuclear abundances and evolution of interstellar medium. Ann. Rev. Astron. Astrophys. 18, p. 399-437.

29. Greenberg J. M. (1984) The structure and evolution of interstellar grains. Sci. Amer. 250, p. 121-135.

30. For a discussion of this question see, Yang J. and Epstein S. (1983) Interstellar organic matter in meteorites. Geochim. Cosmochim. Acta 47 , p. 2199-2216.

31. See, for example, Clayton D. D. (1985) Excess depletion of Al, Ca, and Ti from interstellar gas. This workshop.

32. Donn B. and Scars G. W. (1963) 1. Planets and comets: role of crystal growth in their formation. Science 140, p. 1208-1211.

33. Hayatsu R. and Anders A. (1981) Organic compounds in meteorites and their origins. Topics of Current Chemistry 99, p. 1-37.

34. Fraundorf P., Patel R. I., Walker R. M., Freeman J. J., and Adar F. (1982) Raman spectroscopy of graphite and other phases in meteorites and interplanetary dust. Lunar Planet. Sci. XIII, Houston, TX, p. 225-226.

35. Mogk D. W., Mackinnon I. D. R., and Rictmeijer F. J. M. (1985) Auger spectroscopy of stratospheric particles: the influence of aerosols on interplanetary dust. Lunar Planet. Sci. XVI, Houston, TX, p. 569-570.

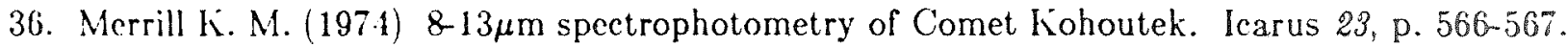

